\title{
Maintaining technical quality of care in the introduction of Cyclofem in a national family planning program: findings from Indonesia
}

\author{
Firman Lubis ${ }^{1}$, Peter Fajans ${ }^{2}$, Ruth Simmons ${ }^{3}$
}

${ }^{1}$ Yayasan Kusuma Buana, Jakarta, Indonesia; ${ }^{2}$ Department of Population Planning and International Health, School of Public Health, University of Michigan, Ann Arbor, MI 48109-2029, USA; and ' Department of Population Planning and International Health and Department of Public Health Policy and Administration, School of Public Health, University of Michigan, Ann Arbor, MI 48109-2029, USA

This paper discusses the technical dimensions of "quality of care" in contraceptive service delivery in both the Cyclofem Introductory Trial, as well as in routine service delivery of other injectables in Indonesia. Although the quality of care in the Cyclofem trial was generally acceptable, substantial weaknesses in screening, clinical technique, the management of side-effects, and knowledge concerning re-injection time frames were identified in the provision of injectable contraceptives in routine service delivery. The findings suggest that in order for Cyclofem and other injectables to be delivered in the routine program with an adequate standard of care, considerable managerial adaptation and strengthening of providers' technical capabilities would be necessary prior to actual introduction. This would include providing training and updated technical guidelines concerning both Cyclofem and other contraceptives to providers, with an emphasis on technical issues including contraceptive indications and contraindications, re-injection timeframes, maintenance of asepsis and the management of side-effects. Strengthening the existing management information system and logistics systems to facilitate differentiation between injectable contraceptives provided by the program so

Address correspondence to: Firman Lubis, YKB, Asem Baris Raya Blok A/3 Tebet, Jakarta Selatan, Indonesia 
as to ensure sufficient supplies of both contraceptives and associated materials such as needles and syringes will also be necessary.

Keywords: monthly combined injectable contraception; Cyclofem; Indonesia; quality of care; family planning; health services delivery

\section{Introduction}

One of the basic strategies of the Indonesian National Family Planning Program is to offer a broad range of appropriate contraceptive options to potential users. This strategy was adopted primarily due to the recognition of both the socio-cultural as well as demographic variation among Indonesian communities. The intent behind this "cafeteria" approach is to allow cach couple to choose the most suitable contraceptive method according to their individual needs. Since the start of the family planning program in 1969, a variety of oral contraceptives, intrauterine devices (IUDs), condoms, injectable contraceptives as well as a major new method, Norplant, have been gradually introduced into the program. Since March 1990, the National Family Planning Coordinating Board (BKKBN) has also been conducting an introductory trial under WHO sponsorship of a once-amonth injectable, Cyclofem, for the purpose of assessing the appropriateness of its addition to the Indonesian program. ${ }^{1-3}$

Interest in Cyclofem arises from the fact that the intrinsic characteristics of this monthly injectable, short duration of action, rapid return of ovulation, more regular bleeding patterns, and high efficacy, make it an especially attractive method from the perspective of young, low parity women who choose to delay the onset of child-bearing or wish to space the timing of births. Focus on young women is a major component of the current strategic plan of the Indonesian National Family Planning Program.

While Cyclofem is attractive in light of the current strategy adopted by $\mathrm{BKKBN}$, it has been realized that addition of a new contraceptive method into ongoing, large and complex programs has extensive implications for program management and quality of carc. The difficulty of assuring appropriate standards in service delivery increases with each method added to the program, making it mandatory to assess whether appropriate capabilities are in place before new contraceptive technology is introduced. Realizing the importance of the management and quality of care implications of new contraceptive technology, BKKBN has conducted health services research in conjunction with the introductory trial of Cyclofem. ${ }^{*}$

\footnotetext{
* Other dimensions of the study, as well as its basic design and methods, are presented in greater detail in the preceding companion piece to this paper "Contraceptive Introduction and the Management of Choice: The Role of Cyclofem in Indonesia" by Simmons et al. ${ }^{5}$
} 


\section{Materials and Methods}

This research study examined the implications for service delivery of adding Cyclofem to the national family planning program. More specifically, it used a quality of care framework to assess the managerial and organizational adaptations that would be necessary to assure quality of care in the introduction of Cyclofem to contraceptive service delivery. ${ }^{6,7}$

This study used a qualitative approach, combining it with secondary quantitative data to the extent that these were available. A qualitative approach was the methodological tool of inquiry most appropriate for the endeavor attempted here because it allowed detailed examination of the management process, and a focus on systems oriented interpretations of program implementation.

Data collection consisted of systematic observation of service delivery, indepth interviews, and document reviews, and was conducted in three provinces, namely, Jakarta, West Java and West Sumatra. Observations were conducted in clinics where Cyclofem was being provided in connection with the introductory trials, as well as in service delivery settings where Cyclofem was not yet available (non-trial health centers).

The non-trial health centers were selected purposely in each province, making an attempt to represent both urban and rural facilities (where appropriate) as well as to include health centers with moderate or average resources and program performance in contrast to the relatively high levels exhibited by the trial health centers.

In total, 28 service delivery sites were observed, including: 4 clinics participating in the introductory trial, 7 non-trial health centers, 4 community-based health posts and 13 private sector settings including both private practices and non-governmental organizations clinics.

Studying these settings allows us to predict what would happen if Cyclofem were introduced in these same delivery contexts. For example, the interpersonal and technical quality of care dimension in the provision of other forms of contraception and in particular other injectables, enables one to predict what will happen with Cyclofem. The characteristics of the service delivery system tend to be similar for a variety of contraceptives. If asepsis is maintained in the delivery of other injectables or IUDs, for example, this provides some ground for anticipating that maintaining asepsis in the provision of injectables might be assured as well.

Service delivery settings in the private sector were also included. An attempt was made to conduct observations in both urban and rural areas, and with a variety of service providers, including nurses, midwives, and doctors.

The instrument for systemizing and recording the results of observations was developed for this study. A key characteristic of the observation technique adopted for this study was its focus on clinic sessions as a whole 
and not solely on individual provider-client interactions. The observation instrument emphasized observation of the patterns whereby clients moved through the clinic or service delivery setting, attempting to understand their experience from the time they approached the service delivery point to their exit from it.

Another characteristic of the observation procedure developed was to document the detailed interactions between provider and client, focusing not only on injectables but on all methods provided during the sessions under observation. Individual interactions between providers and clients were documented both in terms of the exact verbal exchange between them but also in terms of the specific components of service delivery. Typically, observations were conducted by at least two researchers, in most instances involving both a medical doctor and a social scientist.

In-depth interviews were conducted with the following types of respondents:

1. National leaders; in BKKBN, in the Ministry of Health (MOH), from the Indonesian Medical Association, the Indonesian Midwives' Association, the Association of Obstetricians and Gynecologists, the Indonesian Planned Parenthood Association, the Blue Circle Program;

2. BKKBN and $\mathrm{MOH}$ leaders and program managers at the provincial and district level;

3. Public and private sector providers of services in introductory trial, as well as non-trial sites, in urban, semi-urban and rural areas, including BKKBN and $\mathrm{MOH}$ field staff;

4. Community leaders as represented by village leaders, their wives in their capacity as leaders of the PKK, the formal village women's group.

5. Clients, including both Cyclofem acceptors and dropouts in trial areas, and users of other injectables and other methods in non-trial areas.

Interview guidelines for the various categories of respondents were developed to provide a broad outline for the topics to be covered in these in-depth conversations. In-depth interviews were conducted by one or more members of the research team.

\section{Results}

This paper presents findings from the study pertaining to the technical quality of care, with a focus on the medical and technical standards with which contraceptive services are provided. The dimensions of technical quality of care considered here include: (a) assessment of indications and contraindications; (b) clinical technique, especially whether asepsis is maintained in the provision of the "clinical" methods; (c) management of side effects; $(\mathrm{d})$ appropriate follow-up in service delivery. 
Observations of both Cyclofem and other contraceptive service delivery were conducted in health centers participating in the Cyclofem introductory trial, while observations in non-trial health centers included the provision of oral and injectable contraceptives, as well as IUD insertion. These observations are discussed below in terms of the various dimensions of the technical quality of care.

\section{Assessment of Indications and Contraindications}

In the Cyclofem trial, screening for breast-feeding, an important contraindication to the use of estrogen-containing contraceptives, was conducted consistently. Breast-feeding status was typically assessed by providers when women were first queried about their potential interest in joining the trial. However, observation of the formal screening process used later in the assessment of other indications and contraindications of Cyclofem raised several concerns. Appropriate indications, such as the advantages of Cyclofem for women wishing to space their children, were not communicated to women, although occasionally inappropriate indications such as "Cyclofem will make you slender" or "it will help you to gain weight" were used in an attempt to persuade women to enter the trial. In contrast, a series of questions from the trial protocol checklist enumerating contraindications was conscientiously read to each client during enrollment. Although this verbal screening was complete in content, the issue of its potential value or efficacy as a screening tool arose due to the medical terminology used. It is doubtful that clients were able to fully understand the terms used to identify contraindicated conditions. For example, women asked if they suffer from "liver disease, cancer, heart disease, or thromboembolism" are unlikely to either understand or be able to identify the applicability of such terminology to their own condition.

The process of screening observed in the assessment of indications and contraindications for other contraceptives in routine service delivery varied widely from provider to provider, but typical patterns were noted common to both the trial and non-trial health centers. The first question usually asked by providers to clients was the date of their last menstrual period so as to screen for pregnancy. Most women appeared to know that providers typically require a women to be menstruating as a prerequisite for services and thus they come for a family planning method at that time.

The formal screening process is intended to be supported by the use of the so called "K-IV" family planning clinic record form which lists background information for each client including their identity, their reproductive status and desires, and a checklist for the assessment of contraindications. ${ }^{8}$ Direct assessment of indications for the choice of a particular contraceptive based on women's reproductive desires or medical history was only occasionally observed. When women were asked about 
their reproductive intentions, it was usually as a prelude to suggesting the use of a long-acting method such as the IUD. Rarely were younger clients asked about their needs in regard to a contraceptive suitable for spacing. The choice of an appropriate contraceptive depends on a woman's stage in her reproductive life cycle and the K-IV form provides the necessary information, but providers must make use of this information in their assessment. However, this was often not the case.

The section of the K-IV form to be used in screening for contraceptive contraindications is labeled "Screening for Pills", and the screening questions are those appropriate for combined hormonal contraceptives. This is surprising as the National Family Planning Program supplies both combined oral contraceptives, as well as a progestogen-only "mini-pill" for breast-feeding women who desire to use an oral contraceptive. Clearly, the screening form has not been updated since the introduction of this alternative. Perhaps more importantly, the screening checklist provided does not present an appropriate list of contraindications for the two progestogen-only injectable contraceptives provided by the program. It was observed that providers, in general, do not differentiate among the various hormonal contraceptives in the screening process, but instead utilize the checklist intended for combined oral contraceptives for all hormonal contraceptives, including injectables, if and when formal screening does occur. This is a clear example of where the addition of new contraceptive methods without the appropriate adjustment in technical guidelines and directives has resulted in confusion on the part of providers and the inability to maintain technical standards of care.

Moreover, to facilitate the process of screening, an attempt has been made in the K-IV form to translate certain medical contraindications into symptoms that are readily understood by clients. However, some of the symptoms utilized on the form to represent contraindications are so nonspecific that they may unnecessarily prevent women from using the method of their choice. For example, "swollen feet" or "difficulty breathing" are included as signs of heart disease, but these symptoms are very general in nature and may result from a variety of non-contraindicated conditions.

Providers consider a physical exam to check for potential contraindications a prerequisite for each client prior to the adoption of a new contraceptive. Some type of physical assessment was observed to be regularly conducted. However, these exams were extremely variable in content, often consisting of no more than a measurement of the client's blood pressure. Exams also did not focus on the assessment of specific contraindicated conditions (i.e., liver enlargement) but focused more typically on examination for a variety of less sensitive and specific signs. For example, sometimes the client's abdomen would be palpated, but in a manner too superficial to detect pregnancy or other potential anomalies. Perhaps the 
most consistently observed examination for physical signs was an assessment of varicose veins in the lower extremities. Women with even the most minor of superficial varices were often told they could not use a hormonal contraceptive.

The K-IV form is intended to assist the provider in the screening of contraindications, while detailed guidance and instructions in the technical dimensions of service delivery, including the assessment of indications and contraindications for various contraceptives, are contained in the BKKBN Medical Handbook for Family Planning. ${ }^{9}$ A comparison of screening criteria provided by the Medical Handbook with the contraindications for oral contraceptives on the K-IV form shows that the two are not completely congruent. For example, breastfeeding is not mentioned as a contraindication for combined oral contraceptives in the K-IV form, although most health center providers are aware that it is contraindicated. Liver disease, as represented by the symptom of jaundice in the K-IV form is not mentioned in the Handbook, although this too is an important contraindication for oral contraceptives. Perhaps more important was the fact that this medical handbook did not seem to be readily accessible to all providers of clinical family planning methods.

Clearly, the existence of a screening and assessment checklist such as the K-IV form does not assure that an appropriate consideration of indications and contraindications will take place. Yet, if it is to have any impact, it does need to be technically appropriate and to reflect the differences in the indications and contraindications specific to individual contraceptive methods. The fact that many providers ignored the K-IV form may reflect their sense that it is incomplete and inappropriate for many of the methods that they provide. However, it was clear from both interviews with providers and observations that providers' knowledge and actual practice related to the assessment of the indications and contraindications of various contraceptives was weak. The addition of cvery new method that has a slightly different profile of characteristics and contraindications represents additional potential for confusion and poor care if efforts are not made to educate and train providers appropriately. The experience with the Cyclofem trial suggests that providers can take the time to assess contraindications but steps must be taken to assure that screening is effective, rather than either a ritual or a needless barrier to hormonal contraceptive use. ${ }^{10}$

\section{Clinical Technique}

One of the main concerns in clinical technique with regard to contraceptive service delivery is whether or not the maintenance of asepsis is assured. ${ }^{11}$ In the provision of injectables, asepsis must be maintained through appropriate use of sterilized needles and syringes. We observed 
consistent one-time use of disposable syringes and needles in the provision of Cyclofem. In non-trial health centers, we observed that in the service provision of depot-medroxyprogesterone acetate (DMPA) and norethisterone enanthate (NET-EN), disposable needles and syringes were also utilized, but a variety of patterns existed with regard to the maintenance of asepsis. In five of the health centers, disposable needles and syringes were used once and discarded after use. In a second pattern observed in three health centers, providers used the same syringe for five clients, but changed to a new sterile needle for each client. In a third pattern followed in two health centers and one sub-health center, we observed that one syringe and one needle were reused without sterilization for multiple acceptors. In all settings, providers routinely used alcohol to swab the injection site. In one health center, the cotton was used several times, while in all other settings the cotton was used only once. Lack of asepsis in provision of injections clearly presents unacceptable risks for transmission of hepatitis B which is endemic in Indonesia, as well as the potential for HIV transmission once the virus becomes prevalent in the general Indonesian population.

A more general lack of attention to asepsis in contraceptive service delivery was also evident. Only rarely were hands washed before providing services. Running water (either piped or from a bucket with a spigot) was unavailable in the service delivery room in many of the health centers and gloves were regularly reused without proper disinfection. IUDs were observed to be inserted without adequate sterilization of the insertion instruments in several of the settings, and equipment used for pelvic exams sometimes received only cursory cleansing between clients.

\section{Management of Side Effects}

In the Cyclofem trial, information about potential side-effects of the method was generally not communicated directly by providers, but was provided through distribution of a leaflet. Given the low literacy status of many users and the fact that they predominantly speak one of several local languages rather than the national Indonesian language used in the leaflet, many users would not be able to understand these written materials. When discussion of side-effects did occur, it was used more frequently as a motivational strategy rather than as a means of providing women with balanced information about contraceptive use. For example, Cyclofem was promoted to "make your period regular", "make you slim" or "to help you gain weight". When acceptors were observed to complain of potential non-specific side-effects of Cyclofem such as mild aches, pains and occasional dizziness, these complaints tended to be automatically dismissed by providers as inconsequential and not related to contraceptive use. In general, providers did not take the time to assess whether these side-effects were actually related to contraceptive use. 
The two most commonly reported specific side-effects of injectable use were amenorrhea and spotting or irregular bleeding. We observed some complaints about amenorrhea from Cyclofem users, but DMPA users frequently complained of this problem. The first response of the provider appears to be to inform the client that this is nothing to worry about, and that the amenorrhea is normal. In the interviews, providers stated that when amenorrhea persists and the client continues to complain, she may be given one or two cycles of oral contraceptives, and then be switched back to an injectable after menstruating. Several instances of service provision where this was indeed the pattern were observed. When DMPA and NET-EN users complained of spotting or heavy bleeding, providers made little attempt to distinguish the degree of spotting or bleeding or to assess the seriousness of the problem from a medical perspective. Moreover, interviews with providers revealed that their knowledge of how to address these side effects was limited.

\section{Follow-up: Windows for Re-injection}

The typical pattern observed with regard to communication with a client about when to return for re-injection was to tell the woman to come back on a particular date, rather than to inform her of the fact that there is a window of several days during which re-injection could occur. This pattern was the same for all three injectables, DMPA, NET-EN and Cyclofem. Almost all providers of Cyclofem appeared to know that there is a window of time, 30 plus or minus three days, during which Cyclofem can be re-injected. Although some clients knew that there is a time window, providers emphasized one particular day as the appropriate time of return for re-injection. There was considerable disagreement among providers as to the appropriate time window for DMPA re-injection, with the most frequently mentioned periods being either 90 days or twelve weeks. This difference corresponds to a change in the period recommended in the BKKBN technical guidelines which occurred in 1989.9

The pattern of service delivery for women who come for re-injection after the appropriate period of time has elapsed was also variable. Some providers reported that they do not give the injectable, even if the woman is only a day or two past the recommended date of return. In such cases, they switch women to oral contraceptives to await the onset of menstruation, or in some instances ask them to wait for their period without mentioning the use of another contraceptive. Others reported that they inquire about the status of the woman's menstrual cycle and her recent pattern of intercourse to determine whether or not the client might be pregnant. If there is no reason to suspect she might be pregnant, the injection is provided even though the proper window for re-injection has elapsed. 
The main mechanism used to assure return of injectable users at the appropriate time was to write the date on the family planning identification card that each patient keeps. Consistent recording of the return date on the card was observed for both Cyclofem and other injectable clients and women were frequently reminded twice in the course of service delivery to return on the appropriate date. In the Cyclofem trial, a mechanism has been developed for formal follow-up of women who do not return at the appropriate time for their re-injection. This mechanism was established for research purposes, however, and there is no regular system of follow-up for users of other injectable methods if they fail to return for re-injection. Although a variety of approaches to follow-up have been established by field-staff in some individual health centers, the feasibility of these efforts on a more widespread basis is open to question due to a variety of personnel, time and other managerial constraints.

For all practical purposes, women are responsible for remembering the date of their return. We found from interviews with clients that they are very conscientious of this responsibility, often taking special precautions to remember the date. Almost all providers reported that the vast majority of clients return on time and that remembering the date does not appear to be a problem as women mark their wall calendar, a ubiquitous item even in rural households. Providers and clients indicated that Cyclofem is even easier to remember than DMPA, both because it is a shorter 30day period and because clients can be told to come on the same date of each month.

\section{Discussion}

Assuring consistency and technical accuracy in the effort to screen for contraindications, manage side-effects, maintain proper clinical procedures, and provide information and follow-up in a large program with literally thousands of providers is a major undertaking. We observed that under the present system, providers sometimes do not distinguish among hormonal contraceptives in the various dimensions of technical quality of care. Moreover, it is difficult to keep large numbers of providers, both at the health center level and in the decentralized rural delivery system, well intormed about the changes in technical guidelines which occur over time. The changing criteria for technical management resulting from the evolution of hormonal contraceptives is one such example. Key managerial factors which determine or explain the various conditions we observed are listed below:

1. Supply shortages: Chronic shortages of basic equipment and other medical supplies are a major reason for the breakdown in technical quality of care in the delivery of tamily planning and other health 
services. Shortages of syringes and needles are pervasive in the public sector health service delivery system and seriously impair the maintenance of appropriate quality of care in the delivery of injectable contraceptives. In those health centers where a single needle and syringe were used for each client, providers explained that this was possible, despite inadequate supplies provided by the program, because clients were asked either to buy their own syringe and needle or to pay a small charge for the purchase of these supplies.

2. Staff knowledge and attitudes: Inadequate knowledge of the indications and contraindications of specific hormonal and other contraceptives, and a lack of appreciation of the importance of asepsis and the necessary procedures for its maintenance are key reasons for the inadequacies in clinical procedures. Insufficient baseline technical knowledge, lack of recent refresher training, and a lack of accessible written guidelines or instructions may be important factors resulting in these deficits.

3. Patient load: Patient loads have a major influence on the extent to which sterility of equipment and procedures is assured. If many women need services, stocks are quickly exhausted and there is inadequate time between patients to assure appropriate disinfection or sterilization of equipment. Heavy patient loads may also contribute to providers not taking time to systematically screen clients for potential contraceptive contraindications or to provide appropriate physical exams.

4. Provider fears and culture of information-giving: As discussed in the preceding paper by Simmons et al..$^{5}$ information-giving often does not receive much attention in service delivery at the health center. This pattern is particularly strong in regard to the discussion of side-effects as providers often fear that discussion of potential negative consequences may be misinterpreted and discourage contraceptive use.

5. Lack of technical supervision: While administrative supervision is conducted regularly, the technical dimension of supervision is weak. Staff with responsibility for supervision at the district level often lack sufficient technical expertise and are of lower professional status than the physicians in charge of service delivery at the health centers. Thus, issues concerning the technical quality of care being delivered are rarely reviewed or discussed during supervisory visits.

6. General lack of attention to the technical dimensions of quality of care: In the past, the technical dimensions of quality of care in contraceptive service delivery have been de-emphasized in comparison with the attention given to other objectives of the family planning program. Recent BKKBN meetings and workshops have begun to address these issues, but initial attention has focused more on clinical methods including Norplant and IUD insertion and did not include discussion of injectable contraceptives. ${ }^{12}$ 


\section{Program Implications}

The findings presented in this paper document that although some dimensions of the technical quality of care in contraceptive service delivery in the study sites conform to accepted standards, substantial weaknesses in the area of screening, clinical technique, the management of side-effects, and re-injection time frames were also identified. The technical quality of services provided in the context of the Cyclofem introductory trial were considerably stronger, but some weaknesses were observed. These findings suggest that in order for Cyclofem and other contraceptives to be delivered in the routine program with an adequate standard of care, considerable managerial adaptation and strengthening of the technical capabilities of service providers would be necessary. This is particularly evident if one considers that introductory trials are typically conducted in facilities chosen to be representative of a relatively high standard of service delivery.

As these findings are based on observations and interviews in a relatively limited number of study sites, the question must be raised as to how generalizable they are for the Indonesian Family Planning Program as a whole. While the total number of sites is small, the study included four of six health centers participating in the Cyclofem introductory trial. These trial centers were purposively selected by BKKBN to represent strong program management and service delivery capabilities. Non-trial health centers were selected with the assistance of provincial program staff to assure representation of both average and relatively weak service performance. As a result, a broad range of service delivery capabilities were included in this study. The observation of a variety of deficiencies in routine service delivery in both the trial and non-trial health centers suggest that these problems are likely to be common more widely. It is also important to keep in mind that this research is program-based and, thus, many of the issues considered are constant throughout the program rather than variable between health centers. For example, weaknesses in technical guidelines, screening forms, reporting and recording, and logistics systems are likely to be consistent across the national program. Furthermore, these observations were acknowledged and confirmed in interviews with BKKBN and Ministry of Health officials, and documented in other reports. ${ }^{13}$

While some of the observed weaknesses have undoubtedly existed for a long time, many may have become accentuated over the recent years as a variety of new contraceptives have been introduced into the routine program. For example, changes in the composition and range of hormonal contraceptives (including both oral and injectable contraceptives) have resulted in the necessity of increasingly complex criteria for assessing indications and contraindications, re-injection windows, and logistics 
necessary to support service delivery and the management of side-effects. In contrast, the service delivery system may function best with clear, simple rules which can be quickly taught and which are easily understood by both the provider and the client. The search for simplicity and routinized instructions, however, may lead to the use of inappropriate guidelines and compromises in the quality of services provided.

The addition of another hormonal contraceptive method would clearly place an additional burden on the existing health service delivery system and would predictably imply additional confusion and difficulties in the maintenance of the technical quality of care. With specific regard to Cyclofem, it seems plausible to predict that the same weaknesses in technical standards of care that characterize injectable service delivery currently would apply to this newly added contraceptive as well.

On the other hand, one can also view the introduction of Cyclofem as an opportunity for a systematic effort to improve the delivery of injectable services. While it is true that additional contraceptive technology without requisite training and other managerial adaptations would weaken standards of care, it can also be argued that with appropriate and systematic attention to these issues, significant improvements in overall service delivery could be attained. Addition of a new method could provide an opportunity to inform staff about the new method and to update them on the technical requirements for existing methods. Operational problems which currently constitute barriers to the technical quality of care can also be addressed in such training.

If Cyclofem is to be introduced into the family planning program in the public sector, a number of managerial adaptations would appear necessary to achieve and maintain technical quality of care standards. A consideration of our study findings suggest that the following activities or actions would have to be implemented:

1. Technical guidelines and instructions must be updated and made available to all providers. The BKKBN Family Planning Medical Handbook must be updated to include information concerning the various technical dimensions of service delivery in regard to Cyclofem. In particular, Cyclofem must be clearly differentiated from other iniectable contraceptives in regard to its intrinsic characteristics. This handbook should be widely distributed to all service delivery points. In addition, an abbreviated version summarizing the technical guidelines should be distributed to every provider.

2. Training will have to be provided to all family planning service providers in the public sector so as to increase their technical knowledge and skills concerning Cyclofem, in general, and, specifically, its differentiation from other injectables with regard to the various dimensions of technical care. 
3. Written guidelines and other materials should be developed for providers in support of the adequate assessment of the indications and contraindications of Cyclofem and other contraceptives. For example, the contraceptive screening checklist (K-IV) could be adapted so as to include the differing contraindications for each of the available methods. Specific necessary components of an appropriate physical screening exam should also be included.

4. A functional system of routine technical support and supervision for providers must be established.

5. The existing management information system reporting forms should be revised to differentiate Cyclofem from other injectables so that the necessary quantities of needles and syringes can be determined and supplied.

6. The elements of the logistics system related to the supply of both needles and syringes, as well as supply of disinfection solution, should be reviewed so as to determine how best to assure that one disposable needle and syringe be provided for each dose of an injectable contraceptive.

7. The number of clients receiving service delivery of clinical techniques at one place and time should match available staff, the quantity of equipment available and the time necessary for disinfection of equipment prior to reuse.

Clearly this list of managerial and programmatic adaptations to support the introduction of Cyclofem into the national public sector family planning program would present a considerable challenge with regard to both manpower and the financial resources necessary to support these training and logistic requirements. The benefits to the program overall could potentially be significant, particularly if viewed from the perspective that these activities would strengthen the technical quality of care in the provision of a variety of contraceptive methods offered in the Indonesian program. However, prior to commencing with such introduction, program planners must carefully assess whether the advantages offered by the introduction of a new method such as Cyclofem justify the managerial challenge and the financial burden of proceeding. If introduction is contemplated without the managerial adaptations and training discussed above, the result is likely to be further confusion on the part of providers and a concomitant reduction in the overall level of the technical dimensions of quality of care in contraceptive service delivery.

\section{Acknowledgments}

This research was conducted for BKKBN by Yayasan Kusuma Buana (YKB) with tinancial support by the Special Programme of Research, Develop- 
ment and Research Training in Human Reproduction. The authors would like to acknowledge the expert assistance of Dr. Astrid Sulistomo and Ms. Indrawati Subadra in the collection and analysis of the data, as well as the advice and support of Dr. Srihartati Pandi of BKKBN, Dr. Nardho Gunawan of the Ministry of Health and the study steering committee. We would also like to thank Ms. Francis Key, Ms. Suzinne Pak-Gorstein and Ms. Leslie Gray for their editorial assistance in the preparation of the manuscript.

\section{References}

1. World Health Organization, Task Force on Introduction and Transfer of Technologies for Fertility Regulation. Introductory trial of Cyclofem, a oncea-month injectable contraceptive. Assessment of use-effectiveness, continuation rates and reasons for discontinuation-A core protocol. 1991.

2. BKKBN. Hasil penelitian fase pilot suntikan KB Bulanan Cyclofem Propinsi Jawa Barat (Research results of monthly KB Injectable Cyclofem Pilot Phase: West Java Province). Bandung: BKKBN, 1991.

3. BKKBN. Laporan pelaksanaan Cyclofem di DKI Jakarta (Implementation report of Cyclofem in DKI Jakarta). Jakarta: BKKBN, 1991.

4. Lubis F, Fajans P, Sulistomo A, Indrawati S, Simmons R. Service delivery implications of introducing Cyclofem in Indonesia. 1992 (report submitted to WHO and BKKBN).

5. Simmons R, Fajans P, Lubis F. Contraceptive introduction and the management of choice: The role of Cyclofem in Indonesia. Contraception 1994; 49:509-525.

6. Bruce, J. Fundamental elements of the quality of care: A simple framework. Studies in Family Planning 1990;21:61-91.

7. Jain, A. Fertility reduction and the quality of family planning services. Studies in Family Planning 1990;20:1-16.

8. BKKBN. Petunjuk teknis pencatatan dan pelaporan pengendalian lapangan gerakan lkeluarga berencana nasional (Technical guidelines for ficld reporting and recording: National family planning field movement). Jakarta: BKKBN, 1991.

9. BKKBN. Pengayoman medis kcluarga berencana (Family planning medical handbook). Jakarta: BKKBN, 1989.

10. Shelton J, Angle M, Jacobstein, R. Medical barriers to access to family planning. Lancet 1992;340:1334-5.

11. Tietjen L, Cronin W, McIntosh N. Infection prevention for family planning service programs. Durant, OK: Essential Medical Information Systems, 1991.

12. BKKBN. Poko-Poko Arahan Kepala BKKBN Pada Lokakarya Nasional Peningkatan Mutu Pelayanan MKET di Indonesia (The Director of BKKBN's Principle Directives for the National Seminar on the Improvement of Quality of MKET Service Delivery in Indonesia) (Pamphlet), Jakarta: BKKBN, 1991.

13. Ward S, Poernomo SS, Simmons R, Simmons G. Service delivery systems and the quality of care in implementation of NORPLANT in Indonesia. Report submitted to The Population Cunncil, 1990. 